\title{
Hydraulics of braided river dynamics. Insights from flume experiments
}

\author{
Riccardo Vesipa ${ }^{1, *}$, Carlo Camporeale ${ }^{1}$, and Luca Ridolfi ${ }^{1}$ \\ ${ }^{1}$ Department of Environment, Land and Infrastructure Engineering, Politecnico di Torino
}

\begin{abstract}
We focus on the measurement of the topography and bathymetry in flume-models of braided rivers. To this aim, an innovative measurement system is adopted. It consists of a laser-ultrasonic sensor and can survey the bed elevation under flowing water. This measurement system was used to profile a flume transect with a frequency of 2 minutes, without stopping the water discharge. By this technique, the topography and the bathymetry of a single transect was continuously acquired in 3 braided rivers generated with the same experimental set-up. The main results are: (i) the quantification of the variability of the number of channels and number of active channels; (ii) the assessment of the probability distribution of some key hydraulic and morphodynamic parameters; and (iii) the verification of the repeatability of results obtained from flume-models of braided rivers.
\end{abstract}

\section{Introduction}

Braided rivers are complex fluvial systems originated by morphological processes that trigger recurring flow divergences and convergences [1]. As a result, braided rivers present a singlechannel bed that is characterized by the presence of multiple bars becoming visible at lowflow conditions [2]. A key characteristic of braided patterns is the coexistence of several morphological processes that act at very different spatial and temporal scales, and whose interaction is responsible of strong fluctuations in time and space of the the bed-elevation and of the water depth $[3,4]$.

The understanding of braiding dynamics is crucial in many fields, ranging from ecology [5] to engineering [6] and river restoration [7]. In this picture, the measurement of bedelevation and bathymetry is fundamental in a number of issues. Examples are: the evaluation of the sediment discharge [3] and shear stresses [8,9], the study of the effect of vegetation on the geo-morphological evolution of rivers $[10,11]$, the definition of indicators for a robust assessment of the morphological status of rivers [12], and the validation of numerical models [13].

The measurement of the bed-elevation and water depth in braided patterns is a difficult task, not yet fully addressed. In fact, changes of the bed-elevation mainly occur during high water stages and exhibit very short time-scales $[14,15]$. However, during these high stages, bed-elevation surveys are very difficult for technical reasons (e.g., turbidity, wood debris, high velocity of the current). It follows that most of the current quantitative knowledge on

\footnotetext{
*e-mail: riccardo.vesipa@polito.it
} 


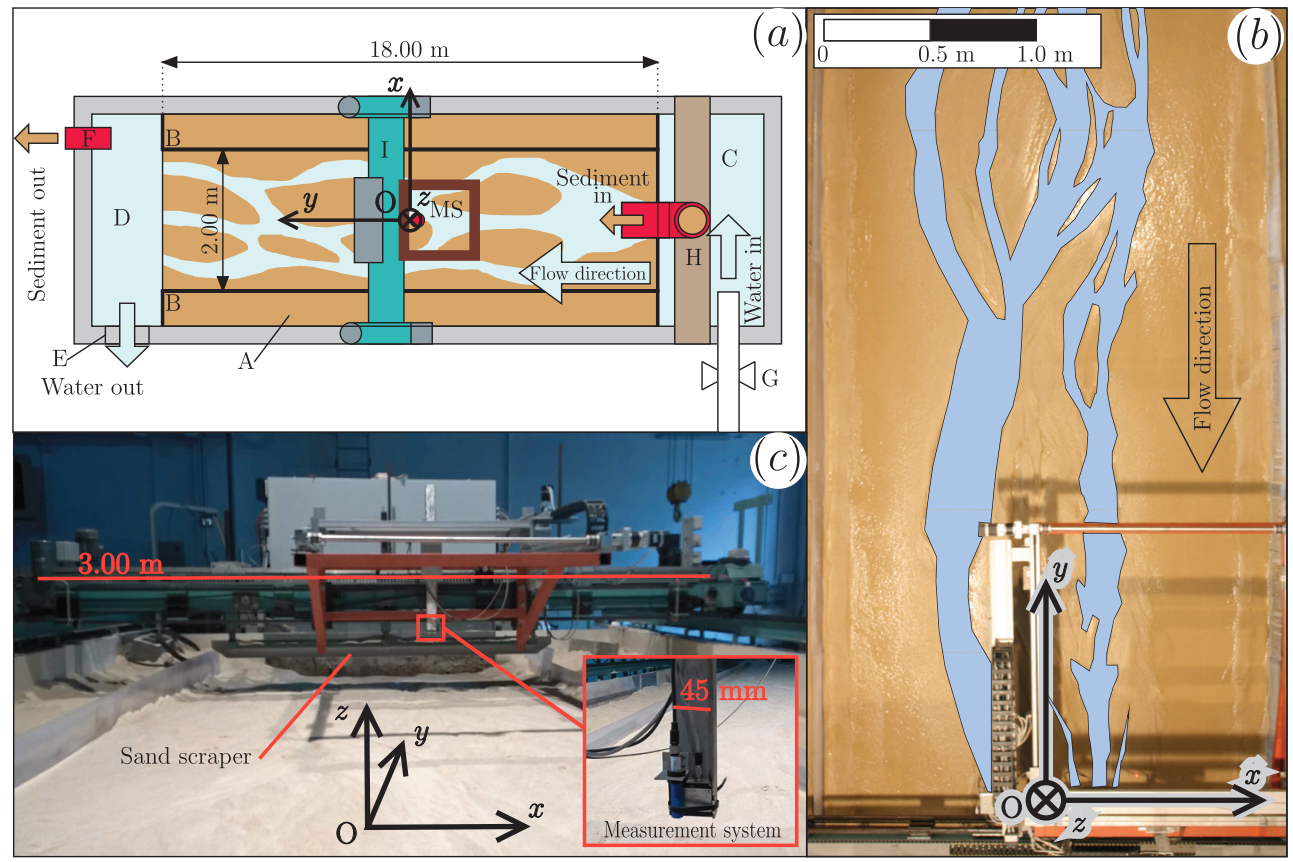

Figure 1. (a) Scheme of the laboratory flume. (b) Close up of the gantry and reference system. (c) Picture of the gantry.

braiding dynamics is based on the measurement of fixed morphologies emerging at low water stages, rather than the measurement of the river dynamics during formative conditions.

The purpose of this work is to study the temporal dynamics of some key hydraulic parameters (e.g., depth and width of channels, shear stresses) observed in flume models of braided rivers. To this aim, we used an innovative experimental apparatus that: (i) measures with very high resolution along the transect (3 points $/ \mathrm{mm}$ ), (ii) measures underwater bed surfaces without stopping the water discharge, and (iii) provides the profile of a river transect and the profile of the water depth with high frequencies (sampling time is 2 minutes). This apparatus was adopted to continuously survey the topography and the bathymetry of a single transect for tens of hours and in 3 braided patterns generated with the same experimental conditions.

The analysis of the collected data allowed to: (i) assess the variability of the number of channels (total and active); (ii) quantify the probability distribution of some relevant hydraulic and morphodynamic parameters; and (iii) verify that results obtained from flume-models of braided rivers are repeatable with runs performed with the same operative conditions.

\section{Methods}

\subsection{Flume set-up}

The experiments were performed in the "G. Bidone" Water Engineering Laboratory at Politecnico di Torino. The flume (A in Figure $1 a$ ) is $18 \mathrm{~m}$ long and $0.6 \mathrm{~m}$ deep. Two side-walls (B) limit the flood plain width to 2 meters. Sand with specific weight of $2650 \mathrm{~kg} / \mathrm{m}^{3}$ and mean grain size $d_{50}=0.45 \mathrm{~mm}$ was used for the experiments (see [15] for further details). In order to provide the prescribed water discharge, the flume is connected to a water stilling tank 
Table 1. Experimental conditions adopted in the three runs performed with the same parameters.

\begin{tabular}{cccccccccc}
\hline$Q$ & $S$ & $Q_{s}$ & $D_{0}$ & $F r_{0}$ & $\beta$ & $\theta_{0}$ & $\mathcal{L}$ & $q$ & $\omega$ \\
\hline$\left(1 \mathrm{~s}^{-1}\right)$ & $(\%)$ & $\left(\mathrm{g} \mathrm{s}^{-1}\right)$ & $(\mathrm{mm})$ & $(-)$ & $(-)$ & $(-)$ & $(\mathrm{mm})$ & $(-)$ & $(-)$ \\
\hline 1.00 & 1.0 & 1.74 & 7 & 1.07 & 70 & 0.096 & 40 & 610 & 6.1 \\
\hline
\end{tabular}

(C). Another tank (D) collects the water and the sediments at the flume end. A moving weir (E) regulates the downstream water level during the experiments, while a siphon (F) removes the sand collected in the tank. The water discharged in $\mathrm{C}$ is regulated by a computerized flow-control-valve (G), and a mechanical hopper (H) equipped with a computer-controlled vibrating panel supplies the upstream bed load.

A key additional equipment is a three-axis $\mathrm{CNC}$ (computer numerical controlled) gantry (I). It carries a scraper and the measurement system (MS) for profiling flume cross sections while water is flowing. The origin of the reference system $\{x, y, z\}$ (point $\mathrm{O}$ in Figure $1 b$ ) is set on the middle of the CNC gantry. The position of the gantry is in the middle of the flume. The gantry can be moved along the $y$ axis. The sand scraper (panel 1c) can be lifted along the $z$ axis to shape the initial channel and floodplain configuration and slope. Finally, the MS can be translated along $x$ with a precision of $\pm 0.5 \mathrm{~mm}$.

\subsection{Runs}

In order to obtain a braided river morphology, the scraper was firstly used to create a floodplain with slope $S$ that accommodated a straight $50 \mathrm{~cm}$ wide rectangular channel. Its depth, $Z$, was chosen to ensure bankfull conditions with the prescribed water discharge $Q[1,4]$. Secondly, the run was started by supplying the liquid discharge $Q$ and the solid discharge $Q_{s}$, where this latter term was calculated by using the results of [16].

Three braided streams were generated in the flume, in order to test the repeatability of results obtained with the same experimental conditions but in different runs. The experimental characteristics of the runs are summarized in Table 1 . Once the channel width $W$, the floodplain slope $S$, and the liquid discharge $Q$ were chosen, the solid discharge $Q_{s}$ and the channel height $Z$ were evaluated to guarantee bed-elevation equilibrium and bankfull conditions, respectively.

To characterize the runs from an hydraulic and morphodynamic viewpoint, the Froude number, $F r_{0}$, the aspect ratio, $\beta$, the Shields stress, $\theta_{0}$, the dimensionless flow rate, $q$, and the dimensionless stream-power, $\omega$, were evaluated as

$$
F r_{0}=\frac{Q}{W \sqrt{g D_{0}^{3}}}, \beta=\frac{W}{D_{0}}, \theta_{0}=\frac{\tau_{0}}{\left(\rho_{s}-\rho\right) g d_{50}}, q=\frac{Q}{\mathcal{L} \sqrt{\Delta g d_{50}^{3}}}, \omega=q \cdot S, \quad(1 a-e)
$$

where $D_{0}$ is the water depth, $\tau_{0}$ is the average bed shear stress, $\rho_{s}$ and $\rho$ are the sediment and water densities, $g$ is gravity, $\Delta=\left(\rho_{s}-\rho\right) / \rho$, and $\mathcal{L}=Q^{2 / 5} / g^{(1 / 5)}$ is a length scale usually adopted to normalize morphometric quantities in braided rivers [4]. The subscripts " 0 " in $(1 a-c)$ denotes that these parameters were computed with reference to the initial rectangular channel. It should also be noted that the adopted experimental conditions are very similar to those adopted in most of the flume studies about braided rivers [4].

\subsection{Profiling of the flume cross section}

During the runs, the bed-elevation of a selected cross section and the position of the free surface of the flowing water were continuously surveyed using a non-intrusive measurement 


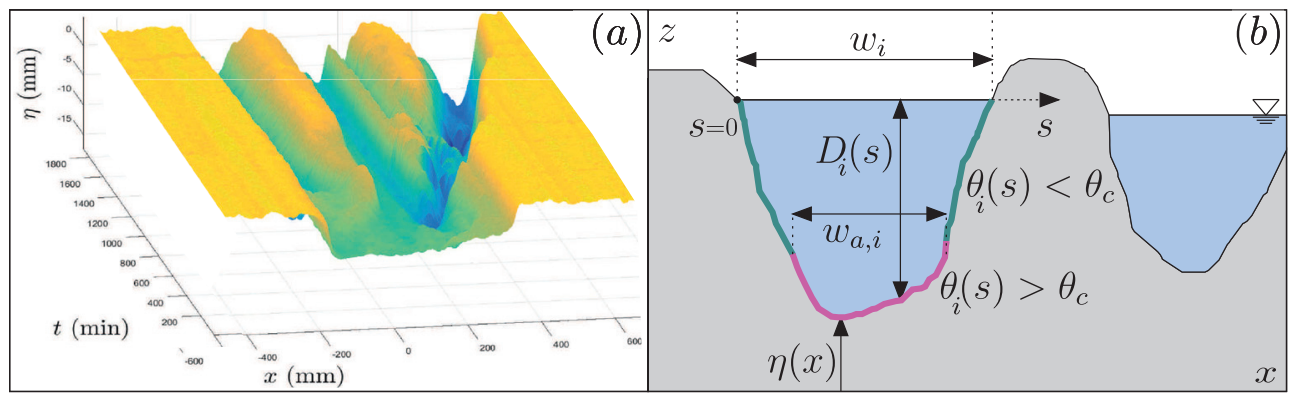

Figure 2. (a) Example of the measured temporal dynamics of a river transect (the surface $\eta\left(x, t_{j}\right)$ is plotted in space and time) for one exemplifying run. For $t \sim 0$ the flume cross section is the rectangular channel carved by the scraper. (b) Parameters evaluated in any single channel for the hydraulic characterization of the river transect.

system (MS) designed by the authors. The MS consists of one laser triangulator and one ultrasonic sensor. It can measure the bed elevation under flowing water as well as the depth of the flowing water. A detailed description and validation of the MS is available in [17] and [15].

The adopted MS provides the elevation of the bed surface, $\eta$, and the water depth, $D$, in a fixed point. Hence, the translation of the MS along the axis $x$ from one bank-side to the opposite bank-side gives the river-transect-profiles of $\eta(x)$ and $D(x)$ at the time $t_{j}$, i.e., $\eta\left(x, t_{j}\right)$ and $D\left(x, t_{j}\right)$. This translation is performed in a time $T_{t}=2 \mathrm{~min}$. Figure $2 a$ reports an example of the three-dimensional picture of the bed topography dynamics of one transect obtained plotting $\eta\left(x, t_{j}\right)$ in space and time, for one exemplifying run.

\subsection{Hydraulic and planimetric parameters}

The data collected by the profiling of the cross section were used to investigate two aspects. The first aspect concerns the study of the morphological activity of the channels. The second aspect is related to the planimetric evolution of the floodplain.

In order to investigate these two aspects, the first preliminary analysis is to detect and isolate every single channel of the river cross section that is observed at the time $t_{j}$. To do this, flooded points $(D(x)>0)$ are detected from the profile of the water depth $D\left(x, t_{j}\right)$. A sequence of flooded points that is delimited by dry points (where $D(x)=0$ ) correspond to a river channel. Channels are then numbered with the index $i=1, \ldots, n\left(t_{j}\right)$, where $n\left(t_{j}\right)$ is the total number of channels observed at the time $t_{j}$.

For every single channel, a new horizontal reference system $s$ is then set (Figure $2 b$ ). The origin of the reference system $(s=0)$ is on the left bank of the river and the direction is toward the right bank. The profile ends at $s=w_{i}\left(t_{j}\right)$, where $w_{i}\left(t_{j}\right)$ is the width of the $i-$ th channel. Every single channel exhibits the depth profile $D_{i}\left(s, t_{j}\right)$, from which the profile of the Shield stress can be estimated from the initial slope of the floodplain, $S$, as

$$
\theta_{i}\left(s, t_{j}\right)=\frac{D_{i}\left(s, t_{j}\right) \cdot S}{\left(\rho_{s} / \rho-1\right) \cdot d_{50}} .
$$

Finally, the evaluation of the zones where the Shield stress exceeds the critical Shield stress (i.e., $\theta_{i}\left(s, t_{j}\right)>\theta_{c}=0.047$, see red curve in Figure $2 b$ ) was performed. This allows to identify the width of the active zone of the $i$-th channel $w_{a, i}\left(t_{j}\right)$. 

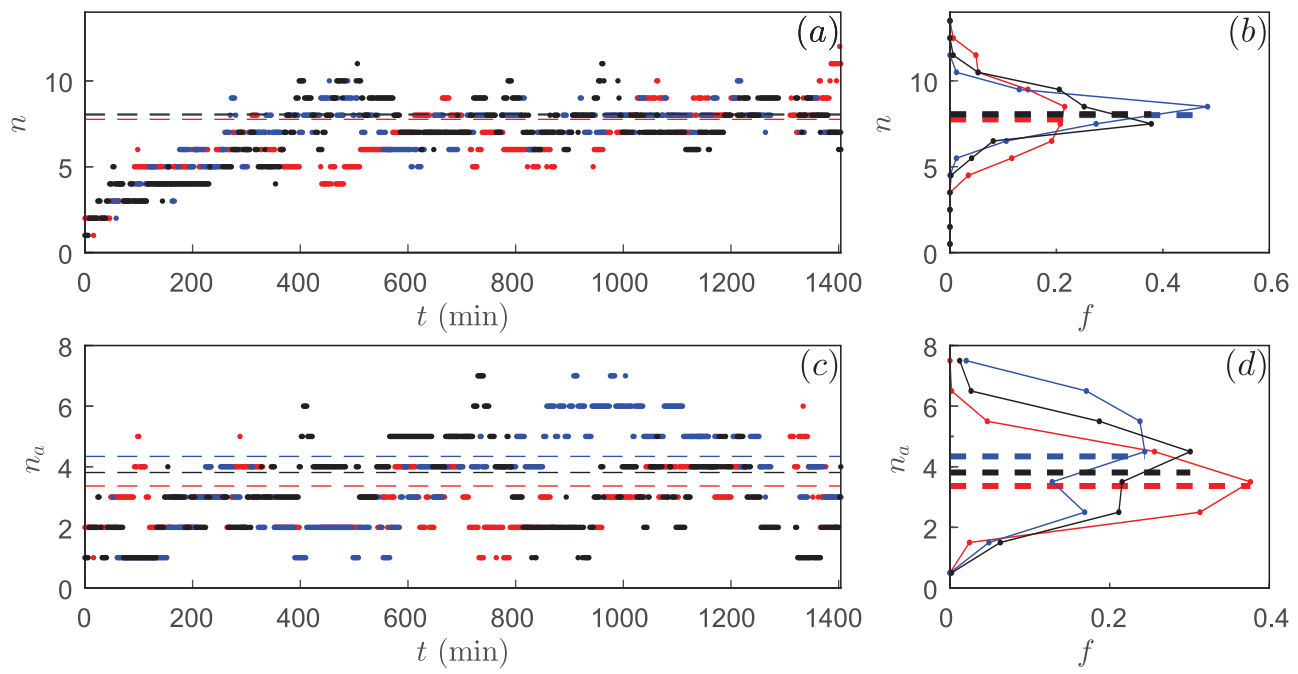

Figure 3. $(a, c)$ Time series of the number of channels, $n$, and number of active channels, $n_{a}$. $(b, d)$ Pdf of the number of channels, $n$, and number of active channels, $n_{a}$. Different colors refer to different runs with the same experimental conditions. The dashed horizontal line is the run-specific mean value of the time series of the considered parameter.

It should be noted that the use of the initial slope of the rectangular channel, $S$, to compute the number of Shields in the fully-formed channels (equation 2) is a crude approximation of the actual local slope. In fact, it is not possible to compute the right amount of sediment feeding that will maintain the channel slope unchanged during the experiments, in particular if the initial channel becomes subdivided in separate channels. A better approach would be considering the final slope of each channel. This could be attained with a new experimental apparatus that monitors the bed topography in two adjacent cross sections.

In order to study the features and the morphological activity of the $i$-th channel over time, four time-dependent scalar parameters are evaluated from the profile $D_{i}\left(s, t_{j}\right)$. These parameters are: the width of the channels, $w_{i}\left(t_{j}\right)$; the maximum water depth, $D_{\max , i}\left(t_{j}\right)=$ $\max \left[D_{i}\left(s, t_{j}\right)\right]$; the mean water depth, $\bar{D}_{i}\left(t_{j}\right)=\left(1 / w_{i}\right) \int_{0}^{w_{i}} D_{i}\left(s, t_{j}\right) \mathrm{d} s$; the percentage of the active zone of the channel $r_{a, i}\left(t_{j}\right)=w_{a, i}\left(t_{j}\right) / w_{i}\left(t_{j}\right)$.

On the other hand, two scalar parameters are adopted to study the planimetric evolution of the river floodplain: the total number of channels, $n\left(t_{j}\right)$; and the number of active channels, $n_{a}\left(t_{j}\right)$. It should be noted that we defined as active the channels where $r_{a}\left(t_{j}\right)>0$.

\section{Results}

\subsection{Planimetric dynamics}

In order to study the dynamics of the floodplain, we focus on the total number of channels and on the number of active channels. Figure $3 a$ reports the time series of the total number of channels, $n\left(t_{j}\right)$ for the three considered runs. According to the reported time series, the evolution of the floodplain can be roughly divided in two phases. During the first phase (from $t_{j}=0$ to $t_{j}=400 \mathrm{~min}$ ), the total number of channels increases from 1 (the initial rectangular channel) to about 7-8. This is a transient phase during which the braided morphology 
is formed [4]. During the second phase (from $t_{j}=400 \mathrm{~min}$ ), the total number of channels fluctuates around 7-8. This is a state of dynamic equilibrium, during which the braided morphology is continuously reworked by the morphological processes but its time-averaged characteristics do not change [4]. The oscillations of the number of channels observed during the dynamic equilibrium are made up of two components. The first component is a significant increment (or reduction) of channels that occurs over long time scales. Consider for instance the run characterized by the red points in Figure $3 a$ : the number of channels grows from 5 to 9 from $t_{j}=500$ min to $t_{j}=700 \mathrm{~min}$ and then reduced from 9 to 5 from $t_{j}=700$ min to $t_{j}=900 \mathrm{~min}$. This remarkable change in the number of channels is probably caused by slow morphodynamic events of high magnitude, such as the transit of large sediment bars. The second component is a very mild oscillations ( \pm 1 channel) that occurs over short time scales ( $\sim 20$ minutes). This moderate alteration is probably induced by fast and minor morphological events (e.g. bifurcation silt-up or chute cutoff). In order to quantify the variability of the number of total channels, the probability density functions (pdf) of the index $n$ is reported in Figure $3 b$. This pdf is evaluated for $t_{j}>400 \mathrm{~min}$, in order to focus on the dynamic equilibrium phase. It can be observed that the pdfs exhibit a clear peak at $n=7-8$ and that the tails extent up to \pm 4 from this most probable value. However, it should be noted that the tails are very thin, and the number of channels most likely to be observed is in the range \pm 2 from the most probable value.

Figure $3 c$ reports the temporal series of the number of active channels, $n_{a}\left(t_{j}\right)$, while the pdfs of $n_{a}$ are reported in Figure $3 d$. Differently from $n, n_{a}$ does not exhibits a marked initial transient phase. In fact, the pdfs computed for $t_{j}>400 \mathrm{~min}$ and for $t_{j}>0 \mathrm{~min}$ basically coincided. The number of active channels grows very fast (from 1 to 3-4) over the first tens of minutes, and then oscillates around this mean value. The pdfs are peaked at $n=3-4$, with a tails up to \pm 4 from the most probable value. Also in this case, tails are very thin, and the most likely number of active channels is in the range \pm 1 from the most probable value.

The comparison of panels $3 a-b$ and $3 c-d$ reveals that $n$ and $n_{a}$ require a very different transient time to reach statistical equilibrium. We believe that this different behavior is due to the different mechanisms that control $n$ and $n_{a}$. The number of active channels is probably governed by the plano-altimetric morphological instability that arise in channels with a high aspect-ratio. When the run is started, multiple bars develop in the flat bottom of the rectangular channel, and quickly divide the channel in 3-4 active channels. The formation of these active channels does not require extensive sediment reworking, and thus can take place rather quickly. On the contrary, the morphological configuration at dynamical equilibrium (about 7 channels) is reached very slowly, as a huge amount of sediments has to be reworked throughout the cross section.

\subsection{Dynamics of hydraulic parameters}

As described in section 2.3, the hydraulic parameters $w_{i}, \bar{D}_{i}, D_{\max , i}$, and $r_{a, i}$ were evaluated for every channel detected at the time $t_{j}$. This procedure was repeated for every cross section sampled after the initial transient described in section 3.1 (i.e., for $t_{j}>400 \mathrm{~min}$ ). This leads to sample sets of $w, \bar{D}, D_{\max }$, and $r_{a}$ that collect the value of the hydraulic parameters of every channel detected over the entire phase of morphological dynamic equilibrium. The analysis of these sample sets of hydraulic parameters is performed through the evaluation of the pdf of such sets (Figure 4).

Panels $4 a-b$ describe the dynamics of the water depth. It can be observed that the experimentally derived distributions of the parameters $D_{\max }$ and $\bar{D}$ resemble a Gamma distribution and that samples exhibit a high variability. In fact, the right tails extend up to 6 times the mean value of the distribution (marked with a thick vertical dashed line). It should also be 

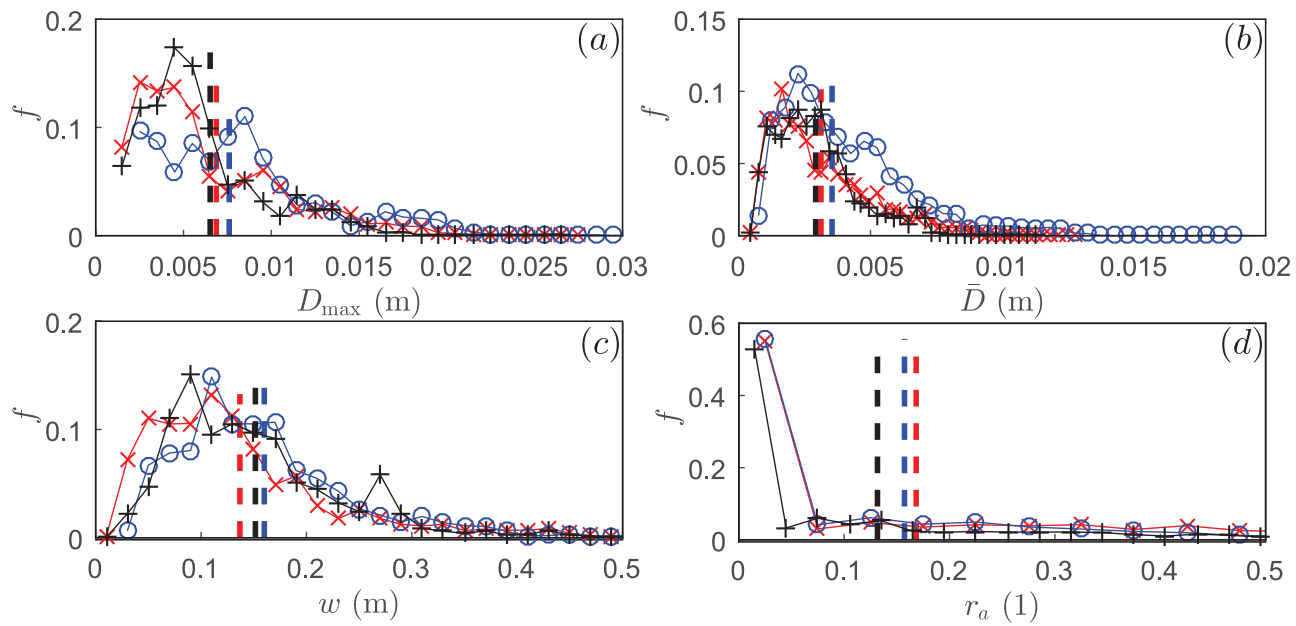

Figure 4. PDFs of some relevant hydraulic parameters. Different colors and markers refer to different runs with the same experimental conditions. The thick dashed vertical line is the mean value of the data-set of the considered parameter.

observed that the maximum depth of the channel, $D_{\max }$, is on average about two times the mean value, $\bar{D}$. This means that channels exhibit a strong variability of the water depth along the transversal direction $s$. Thanks to the proportionality relation (2) between $D(s)$ and $\theta(s)$, panels $4 a-b$ allows also the analysis of the probability distribution of shear stresses in the channels. Also the shear stresses exhibit a high variability, and the maximum Shield stress $\theta_{\max }$ is about two times the mean Shield stress $\bar{\theta}$. This means that a high variability of morphological activity is observed along the transversal direction of braided river channels. This suggests that preferential patterns of localized sedimentation/erosion inside channels may lead to the fast formation of lateral or central sediment bars.

Finally, the measured dynamics of the total width and active width of channels is reported in panels $4 c-d$. Similar to the parameters discussed before, also the width of channel exhibits a distribution that resemble the Gamma distribution, and is characterized by a high variability. Differently, the ratio $r_{a}$ exhibits a peculiar behavior, with a very marked peak at $r_{a} \sim 0.05$, and a wide variability (flat curve) in the range $[0.1,0.5]$. From a morphodynamic point of view, this distributions entails that most of the channels exhibit a very weak and localized morphological activity $\left(r_{a}=w_{a} / w\right.$ is only $\left.5 \%\right)$. On the other hand, the active width of channels that are active exhibits a high variability. Interestingly, no channels with more than $50 \%$ of active width $\left(r_{a}>0.5\right)$ were observed.

\subsection{Repeatability of results}

In this paper we reported for every pdf or time-series the results obtained from three different runs performed with the same operative and experimental conditions. This allowed us to test the repeatability of the results obtained from flume-experiments of braided rivers. It can be noticed that the time series of $n$ and $n_{a}$ (panels $3 a-c$ ) are very similar and exhibit the same trend. In particular, in every run, a dynamic equilibrium is reached after about $400 \mathrm{~min}$. The analysis of the three pdfs derived from the time series of $n$ and $n_{a}$ reported in panels $3 b-d$ reveals also that the variability of number of channels is the same in any run. The repeatability of the results is confirmed also for the probability distribution of the data sets of 
the parameters $w, \bar{D}, D_{\max }$, and $r_{a}$. In fact, the run-specific pdfs (pdfs with different colors) in Figure 4 qualitatively overlap very well, exhibit the same most probable values, and the tails extent is basically the same.

\section{Conclusion}

We have studied the planimetric and hydraulic temporal dynamics of braided-networks in flume experiments. To this aim, we adopted an experimental technique that allowed us the detailed measurement of the bed-elevation and water depth of a flume transect with a minimum sampling time of 2 minutes. Such a short sampling time was possible because the survey of the bed-elevation did not require the water discharge to be stopped. Three runs were performed with the same experimental conditions, in order to test the repeatability of results obtained from different runs. The main results were: (i) to determine the variability of the number of total channels and active channels; (ii) to quantify the probability distribution of some important morphodynamic parameters; and (iii) to assess that flume-models of braided rivers give repeatable results, if runs are performed with the same operative conditions.

Future analyses will study the effect of the experimental parameters (e.g., $q$ and $\omega$ ). These analyses may help in increasing the understanding of the physical mechanisms responsible for the formation of braided morphologies.

\section{Acknowledgments}

We would like to thank Matteo Buschini, Jan Sabbagh and Gianluca Siddi for their precious help in running the experiments.

\section{References}

[1] P. Ashmore, Can. J. Earth. Sci. 28, 326 (1991)

[2] P. Ashmore, in Treatise on Geomorphology (Elsevier Inc., 2013), Vol. 9, pp. 289-312

[3] D. Milan, G. Heritage, D. Hetherington, Earth Surf. Processes Landf. 32, 1657 (2007)

[4] W. Bertoldi, L. Zanoni, M. Tubino, Earth Surf. Processes Landf. 34, 547 (2009)

[5] R. Beschta, W. Ripple, Geomorphology 157-158, 88 (2012)

[6] H. Piégay, G. Grant, F. Nakamura, N. Trustrum, in Braided Rivers (Blackwell Publishing Ltd., 2009), pp. 257-275

[7] A. East, G. Pess, J. Bountry, C. Magirl, A. Ritchie, J. Logan, T. Randle, M. Mastin, J. Minear, J. Duda et al., Geomorphology 228, 765 (2015)

[8] C. Paola, in Coherent flow structures in open channels, edited by P. Ashworth, S. Bennett, J. Best, M. S. (John Wiley \& Sons, 1996)

[9] A. Nicholas, Geomorphology 36, 89 (2000)

[10] W. Bertoldi, N. Drake, A. Gurnell, Earth Surf. Processes Landf. 36, 1474 (2011)

[11] R. Vesipa, C. Camporeale, L. Ridolfi, Geophys. Res. Lett. 42, 8021 (2015)

[12] M. Redolfi, M. Tubino, W. Bertoldi, J. Brasington, Water Resour. Res. 52, 5951 (2016)

[13] R.D. Williams, J. Brasington, D.M. Hicks, Geogr. Compass 10, 102 (2016)

[14] J. Lindsay, P. Ashmore, Earth Surf. Processes Landf. 27, 27 (2002)

[15] R. Vesipa, C. Camporeale, L. Ridolfi, Water Resour. Res. 53, 5184 (2017)

[16] W. Bertoldi, P. Ashmore, M. Tubino, Geomorphology 103, 330 (2009)

[17] F. Visconti, L. Stefanon, C. Camporeale, F. Susin, L. Ridolfi, S. Lanzoni, Earth Surf. Processes Landf. 37, 818 (2012) 